\title{
Correction to: Mild TBI Results in a Long-Term Decrease in Circulating Phospholipids in a Mouse Model of Injury
}

\author{
Tanja Emmerich 1,2,3 $\cdot$ Laila Abdullah ${ }^{1,2,3} \cdot$ Joseph Ojo $^{1,2} \cdot$ Benoit Mouzon $^{1,2,3} \cdot$ Thinh Nguyen $^{1} \cdot$ Gogce Crynen $^{1,2}$. \\ James E. Evans ${ }^{1,3}$. Jon Reed ${ }^{1,3} \cdot$ Michael Mullan $^{1} \cdot$ Fiona Crawford ${ }^{1,2,3}$
}

Published online: 20 February 2020

๑) Springer Science+Business Media, LLC, part of Springer Nature 2020

\section{Correction to: Neuromol Med (2017) 19:122-135 https://doi.org/10.1007/s12017-016-8436-4}

The original version of this article unfortunately contained a mistake. Gary S. Laco should not be listed as an author in the author group.

The original article can be found online at https://doi.org/10.1007/ s12017-016-8436-4.

Tanja Emmerich

temmerich@roskampinstitute.net

Laila Abdullah

labdullah@roskampinsitute.net

Joseph Ojo

bojo@ roskampinstitute.net

Benoit Mouzon

bmouzon@rfdn.org

Thinh Nguyen

tnguyen@roskampinstitute.net

Gogce Crynen

gkayihan@rfdn.org

James E. Evans

jevans@ roskampinstitute.net
Publisher's Note Springer Nature remains neutral with regard to jurisdictional claims in published maps and institutional affiliations.
Jon Reed

jreed@rfdn.org

Michael Mullan mmullan@rfdn.org

Fiona Crawford fcrawford@rfdn.org

1 The Roskamp Institute, 2040 Whitfield Avenue, Sarasota, FL 34243, USA

2 The Open University, Walton Hall, Milton Keynes, Buckinghamshire MK7 6AA, UK

3 James A. Haley Veteran's Hospital, 13000 Bruce B. Downs Blvd., Tampa, FL 33612, USA 